\title{
Online MR image reconstruction for compressed sensing acquisition in $\mathrm{T}^{*}$ imaging.
}

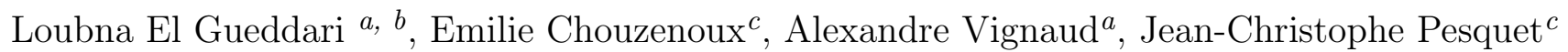 \\ and Philippe Ciuciu ${ }^{a, b}$ \\ ${ }^{a}$ CEA/NeuroSpin, Bât 145, F-91191 Gif-sur Yvette, France. \\ ${ }^{b}$ Inria-CEA Saclay Ile-de-France, Parietal team, Univ. Paris-Saclay, France. \\ ${ }^{c} \mathrm{CVN}$, Inria Saclay, Centrale-Supélec, Univ. Paris-Saclay, France.
}

\begin{abstract}
Reducing acquisition time is a major challenge in high-resolution MRI that has been successfully addressed by Compressed Sensing (CS) theory. While the scan time has been massively accelerated by a factor up to 20 in $2 \mathrm{D}$ imaging, the complexity of image recovery algorithms has strongly increased, resulting in slower reconstruction processes. In this work we propose an online approach to shorten image reconstruction times in the CS setting. We leverage the segmented acquisition in multiple shots of k-space data to interleave the MR acquisition and image reconstruction steps. This approach is particularly appealing for $2 \mathrm{D}$ high-resolution $\mathrm{T}_{2}^{*}$-weighted anatomical imaging as the largest timing interval (i.e. Time of Repetition) between consecutive shots arises for this kind of imaging. During the scan, acquired shots are stacked together to form mini-batches and image reconstruction may start from incomplete data. For each newly available mini-batch, the previous partial solution is used as warm restart for the next sub-problem to be solved in a timing window compatible with the given TR and the number of shots stacked in a mini-batch. We demonstrate the interest and time savings of using online MR image reconstruction for Cartesian and non-Cartesian sampling strategies combined with a single receiver coil. Next, we extend the online formalism to address the more general multi-receiver phased array acquisition scenario. In this setting, calibrationless image reconstruction is adopted to remain compatible with the timing constraints of online delivery. Our retrospective and prospective results on ex-vivo $2 \mathrm{D} \mathrm{T}_{2}^{*}$-weighted brain imaging show that high-quality MR images are recovered by the end of acquisition for single receiver acquisition and that additional iterations are required when parallel imaging is adopted. Overall, our approach implemented through the Gadgetron framework may be compatible with the data workflow on the scanner to provide the physician with reliable MR images for diagnostic purposes.
\end{abstract}

Keywords: C ompressed Sensing; Magnetic Resonance Imaging; Image reconstruction; Online processing; Structured sparsity.

\section{INTRODUCTION}

Magnetic Resonance Imaging (MRI) is a key medical imaging technique to non-invasively probe soft-tissues with no side effects. The main limitation of MRI is its long acquisition time, especially in the high-resolution (i.e. sub-millimetric) imaging context. Indeed, the short lifespan of the MR signal makes the spatial encoding in large k-spaces (i.e. high-resolution setting) necessarily segmented. MR imaging also provides a wide range of contrasts (such as $\mathrm{T}_{1}, \mathrm{~T}_{2}, \mathrm{~T}_{2}^{*}$, etc.), allowing the physician to look at different tissue characteristics of any organ under investigation. The so-called $\mathrm{T}_{2}^{*}$-weighting contrast refers to the observation of $\mathrm{T}_{2}^{*}$ relaxation effect, produced by the decay of transverse magnetization. From a medical diagnostic perspective, the $\mathrm{T}_{2}^{*}$ contrast is used to detect paramagnetic lesions that characterize brain pathologies such as cerebral hemorrhage or arteriovenous malformation. ${ }^{1}$ This contrast is associated with long echo and repetition times (TE and TR, respectively) ${ }^{2}$ and classically implemented in a gradient recalled echo pulse sequence.

Many strategies have been devised to reduce MR acquisition times while preserving image quality, such as partial Fourier, ${ }^{3}$ parallel imaging ${ }^{4-6}$ and later on compressed sensing ${ }^{7,8}$ (CS). The latest framework is known to achieve higher acceleration factors especially when combined with non-Cartesian sampling strategies such as radial, variable density spiral, ${ }^{9}$ or the recently proposed Sparkling trajectories. ${ }^{10}$ However, due to the presence 
of non-uniform ${ }^{11}$ or non-equispaced ${ }^{12}$ Fourier transform in the forward model, non-Cartesian CS imaging may lead to even longer image reconstruction times as compared to Cartesian CS imaging. Depending on spatial resolution and the number of receivers in the coil, such computational load may become incompatible with the online examination and fast quality check of MRI scans (e.g. check the presence of motion artifacts or any suspicious abnormalities) performed by the MR technician. Too slow MR image reconstruction may also prevent any update of the imaging protocol prescribed by the physician such as the inclusion of a new pulse sequence with potential injection of paramagnetic contrast agent (e.g. gadolinium) that permits to boost the signal-to-noise ratio (SNR).

In this work we propose a new way of accelerating MR image reconstruction in the context of CS-accelerated acquisitions. Instead of performing offline image reconstruction by minimizing a sparsity promoting regularized objective function as most of existing works $8,13,14$ do, we introduce an online image reconstruction approach so that acquisition and reconstruction processes become interleaved. The ultimate goal is actually to reduce the cumulative time of acquisition and reconstruction. To this end, we adopt a mini-batch formalism, that consist of stacking together collected k-space samples to form a so-called mini-batch. Once a mini-batch is available, the reconstruction algorithm is run from an incomplete k-space data set for a few iterations. Hereafter, the current image solution is then used as a warm restart for the next mini-batch processing. This new framework allows us to reach a decent image quality by the end of acquisition. This partial image might be used as a first quality control performed by the technician to accept or relaunch the exam. This novel reconstruction framework can be interfaced with the Gadgetron ${ }^{15}$ project and thus display the resulting images reconstruction directly to the MR system console, hence making CS implementations available beyond any vendor solution.

The rest of the paper is organized as follows. We first set the mathematical formalism and derive two use cases: the first one is simpler and corresponds to the single receiver coil. Although of pedagogical interest, its practical application is limited for high-resolution imaging as we need multiple receivers coil to boost the input SNR in this context. For that reason, the online formalism is then extended to deal with multiple-channel coil and actually combined with calibrationless image reconstruction. ${ }^{16}$ Next, the new algorithm is presented and the setting of its hyper-parameters and mini-batch size is discussed. The proposed online reconstruction method is evaluated on retrospective Cartesian sampling as well as on prospective non-Cartesian k-space data collected. Ex-vivo brains were scanned using Sparkling trajectories at $7 \mathrm{~T}$ for $2 \mathrm{D}$ high-resolution $(400 \mu \mathrm{m}) \mathrm{T}_{2}^{*}$-weighted imaging using single and multi-receiver array coils.

\section{Notation and definitions}

In the following, we will denote vectors with bold letters, e.g. $\boldsymbol{v}=\left[v_{1}, \ldots, v_{p}\right]^{\top} \in \mathbb{C}^{p}$ a $p$-size complex-valued vector. We define by $n$ the target image resolution and $N=n^{2}$ the image or slice dimensions (e.g. $n=512$ while $N=512^{2}=262,144$ pixels). Matrices are denoted by bold upper case letters (e.g., $\boldsymbol{A}$ ). The transpose of a matrix $\boldsymbol{A}$ is denoted by $\boldsymbol{A}^{\top}$, its Hermitian transpose by $\boldsymbol{A}^{\dagger}$, its spectral norm by $\|\boldsymbol{A}\|$, and its Frobenius norm by $\|\boldsymbol{A}\|_{\mathrm{F}}$.

We denote by $\Gamma_{0}\left(\mathbb{C}^{p}\right)$ the set of convex, proper, lower semi-continuous functions on $\mathbb{C}^{p}$ taking values on $\mathbb{R} \cup\{+\infty\}$. The proximity operator at $\boldsymbol{z} \in \mathbb{C}^{p}$ of a function $g \in \Gamma_{0}\left(\mathbb{C}^{p}\right)$ is defined as: ${ }^{17} \operatorname{prox}_{g}(\boldsymbol{z})=\underset{\boldsymbol{v} \in \mathbb{C}^{p}}{\arg \min } \frac{1}{2} \| \boldsymbol{z}-$ $\boldsymbol{v} \|^{2}+g(\boldsymbol{v})$.

\section{PROBLEM STATEMENT}

In this work we propose to take advantage of the sequential aspect of segmented acquisition to start the reconstruction process during dead times in acquisition (i.e. TR) associated with the timing separating consecutive shots. Hence, the two processes get interleaved and partial feedback may be delivered to the MR technician during the scan. Two cases will be studied hereafter. First, we will assume the use of a single-channel receiver coil. Though simpler, this case is rarely met in practice in the high resolution context. To boost the input SNR, multiple receiver coils are traditionally used. The second investigated case thus corresponds to online reconstruction from a multi-channel receiver coil acquisition. In this setting, state-of-the art methods often rely on the coil sensitivity information that requires an estimation step for each scan. To overpass this difficulty, we will rely on a recently proposed calibrationless method ${ }^{16}$ method that tackles the image reconstruction problem with the great advantage of not requiring the knowledge of sensitivity matrices. 


\subsection{General online problem statement}

Acquisition considerations. In segmented acquisition, multiple radio-frequency (RF) pulses are used to tip the global magnetization of a given slice (2D imaging) or volume (3D imaging). More precisely, at each repetition time (TR), a new RF pulse is delivered and a new shot is collected [18, Chap.11 2.2]. Hence, multiple shots are used to collect portions of k-space associated with a given field of view. The k-space samples of a given shot are acquired over a continuous curve driven by the spatial encoding gradient profiles, the so-called k-space trajectory. For example, parallel straight lines are mostly used for Cartesian sampling, oblique lines crossing the k-space center (also called spokes) form Radial trajectory and Archimedean spirals may be used in nonCartesian acquisitions. These non-Cartesian trajectories offer stronger robustness to motion sensitivity although they are prone to off-resonance effects (susceptibility artifacts) and trajectories errors. In the CS setting, one key ingredient to massively undersample the $\mathrm{k}$-space and speed up the acquisition lies in choosing a good variable density ${ }^{19,20}$ that samples more often the low frequencies as compared to the high ones. From a theoretical ${ }^{20,21}$ and practical ${ }^{8}$ viewpoint, power law decaying radial densities are good candidates.

In the following, $S$ represents the number of shots used to fill the k-space hence, the total scan time is equal to $S \times \mathrm{TR}$. For each $i \in\{1, \ldots, S\}$, we refer to the k-space support of the $i^{t h}$-shot as $\Gamma_{i}$ and $\boldsymbol{y}_{\Gamma_{i}} \in \mathbb{C}^{C}$ gathers $C$ samples measured over this shot. Next, let $k \in\{1, \ldots, S\}$, we define $\Omega_{k}$ as the concatenation of the $k$ first collected shots. Hence $\Omega_{k}=\cup_{i=1}^{k} \Gamma_{i}$, and the k-space measurements associated with $\Omega_{k}$ read $\boldsymbol{y}_{\Omega_{k}} \in \mathbb{C}^{k C}$. Using the aforementioned notation, we define the acceleration and under-sampling factors (AF and UF, respectively) with respect to the Cartesian reference as the ratios $n / S$ and $N /(S C)$, respectively. Note that in the CS context, we get both $S<n$ and $S C<N$. Although AF and UF usually evolve similarly (i.e. $\mathrm{AF}=\mathrm{UF}$ ), the recently introduced Sparkling sampling scheme ${ }^{10}$ breaks down this relation as AF $>$ UF.

Reconstruction. In standard offline approaches, image reconstruction from the k-space data is performed by minimizing an objective function that sums a data consistency term $f_{\Omega_{S}}$ (depending on the trajectory support $\Omega_{S}$ ) and a regularization term $g$, which usually promotes sparsity in a given multiscale decomposition $\Psi \in \mathbb{C}^{N_{\Psi} \times N}$ such as a wavelet transform. ${ }^{22}$ The general offline image reconstruction problem aims at finding:

$$
\widehat{\boldsymbol{x}} \in \underset{\boldsymbol{x} \in \mathbb{C}^{N}}{\arg \min } f_{\Omega_{S}}(\boldsymbol{x})+g(\boldsymbol{\Psi} \boldsymbol{x}),
$$

where the following standard assumptions are made: (i) $g \in \Gamma_{0}\left(\mathbb{C}^{N_{\Psi}}\right)$ with a closed form proximity operator, and (ii) $f_{\Omega_{S}}$ is convex, differentiable on $\mathbb{C}^{N}$ and its gradient $\nabla f_{\Omega_{S}}$ is $\beta_{S}$-Lipschitz i.e.

$$
\left(\forall\left(\boldsymbol{x}, \boldsymbol{x}^{\prime}\right) \in \mathbb{C}^{N}\right) \quad\left\|\nabla f_{\Omega_{S}}(\boldsymbol{x})-\nabla f_{\Omega_{S}}\left(\boldsymbol{x}^{\prime}\right)\right\| \leq \beta_{S}\left\|\boldsymbol{x}-\boldsymbol{x}^{\prime}\right\| .
$$

Problem (1) can be efficiently solved using iterative majorize-minimze ${ }^{23}$ optimization or proximal methods such as forward-backward splitting algorithm ${ }^{24}\left(\right.$ ISTA $^{25}$ or one of its acceleration FISTA ${ }^{26}$ or POGM $^{27}$ ) or primaldual approaches (ADMM, ${ }^{28}$ Douglas-Rachford, ${ }^{29}$ Chambolle-Pock, ${ }^{30}$ Condat-Vư ${ }^{31,32}$ etc.). Note that for overcomplete dictionaries ${ }^{33}$ the use of primal-dual approaches is more efficient in terms of computation time since these methods do not require the use of an inner iterative solver ${ }^{24}$ to compute the proximity operator of $g \circ \Psi$.

For online reconstruction, the data-consistency term is progressively filled during the scan and reconstruction starts with incomplete data. Concretely, incomplete versions of Problem (1) are solved in order to compute:

$$
\widehat{\boldsymbol{x}}_{k} \in \underset{\boldsymbol{x} \in \mathbb{C}^{N}}{\arg \min } f_{\Omega_{k}}(\boldsymbol{x})+g(\boldsymbol{\Psi} \boldsymbol{x}),
$$

with $k$ the number of available shots so far, and $\widehat{\boldsymbol{x}}_{k}$ the resulting solution, an approximation of which is used as initialization for the next shot processing. In basic online reconstruction techniques, the shots are processed one by one (i.e., $k$ is incremented by one at each step). However, such strategy is only feasible in practice if TR is 
longer than the time $T_{\text {it }}$ needed to solve SubProblem (3), which is rarely met in practice. In particular, in multichannel non-Cartesian reconstruction, the time per iteration is longer than TR, therefore, we adopt a mini-batch formulation presented in Algorithm 1 where $b_{s}$ consecutive spokes are stacked together to form a mini-batch. Then, a warm restart strategy is implemented once an complete mini-batch is available, i.e. SubProblem (3) is not solved for any integer $k$ but only for multiples of $b_{s}$. In this setting, the maximum number of iterations $n_{b}$ per subproblem in Algorithm 1 is thus given by:

$$
n_{b} \times T_{\mathrm{it}} \approx b_{s} \times \mathrm{TR}
$$

In Algorithm 1, the result of $n_{b}$ iterations of the chosen proximal algorithm for solving SubProblem (3), initialized with $\boldsymbol{z}_{0}$, is denoted by $\mathcal{A}_{k, n_{b}}\left(\boldsymbol{z}_{0}\right)$. The variable $\boldsymbol{z}_{k}$ includes an approximation $\boldsymbol{x}_{k}$ to $\widehat{\boldsymbol{x}}_{k}$, but it may also include additional information e.g. a dual variable delivered by the optimization algorithm. Note that when the last mini-batch is acquired $(k=S), n_{b}$ must be relaxed and set large enough to ensure convergence. The basic online formulation is recovered for a batch size $b_{s}=1$.

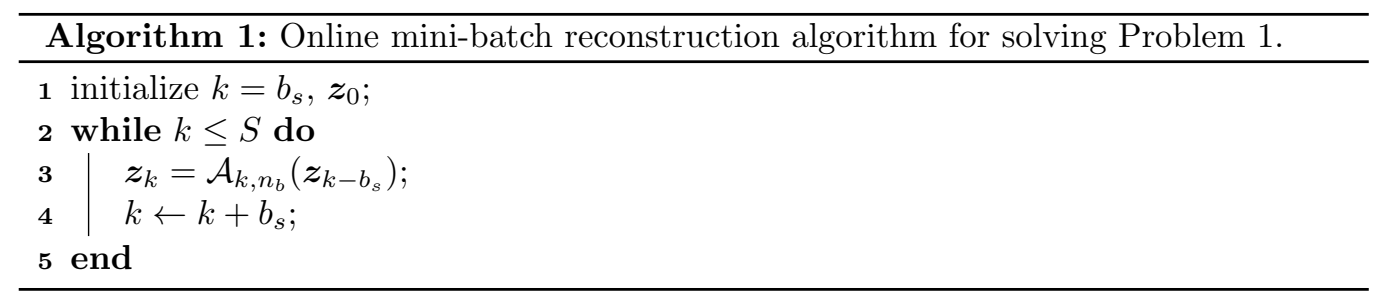

It is worth noting that, since SubProblem (3) is convex, any good optimization algorithm is guaranteed to converge to a global solution whatever its initialization. However, the time to converge to the solution may be very sensitive to the initial guess. Therefore the warm restart procedure is beneficial to reduce the overall reconstruction time. To solve Subproblem (3), we propose to make use of the primal-dual Condat-Vù ${ }^{28,32,34}$ approach summarized in Algorithm 2. Note that $\beta_{k}$ denotes here the Lipschitz constant of $\nabla f_{\Omega_{k}}$. According to [34, Theorem 3.1], Algorithm 2 converges to a solution of SubProblem (3).

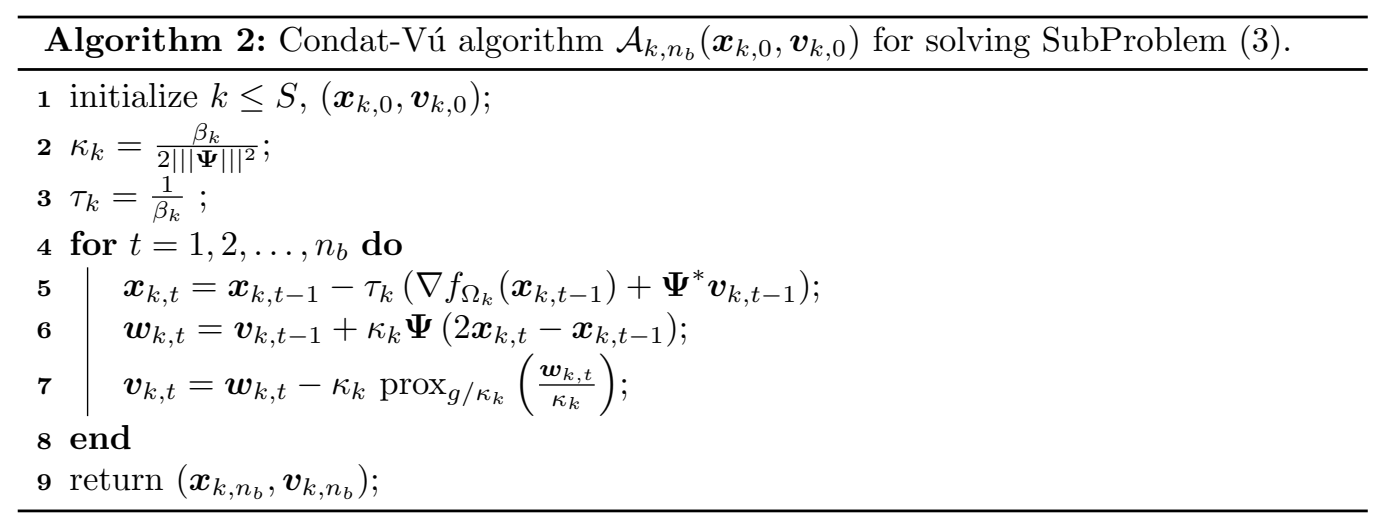

In Section 3.2 we will discuss the choice of the mini-batch size $b_{s}$ and the setting of hyper-parameters. Hereafter, we first derive the reconstruction problem for the single receiver channel coil acquisition before analyzing the more complex multi-receiver coil acquisition setup.

\subsection{Single-channel receiver coil}

In the single-channel context, Algorithm 2 is applied to the following mini-batch formulation of SubProblems (3):

$$
(\forall k \in\{1, \ldots, S\}) \quad \hat{\boldsymbol{x}}_{k}=\underset{\boldsymbol{x} \in \mathbb{C}^{N}}{\arg \min } \frac{S}{2 k}\left\|\boldsymbol{F}_{\Omega_{k}} \boldsymbol{x}-\boldsymbol{y}_{\Omega_{k}}\right\|_{\mathrm{F}}^{2}+\lambda\|\boldsymbol{\Psi} \boldsymbol{x}\|_{1},
$$


where $\lambda$ is the positive hyper-parameter that controls the sparsity level and can be efficiently set in an offline manner. In the case of Cartesian acquisition, the following relation describes the operator $\boldsymbol{F}_{\Omega_{k}}=\Omega_{k} \boldsymbol{F}$ where $\Omega_{k}$ is the under-sampling binary mask and $\boldsymbol{F}$ the fast Fourier transform (FFT). In the case of non-Cartesian acquisition, $\boldsymbol{F}_{\Omega_{k}}$ refers to non-equispaced or non-uniform FFT. ${ }^{11,12}$ While the Lipschitz constant is $\beta_{k}=S / k$ for Cartesian acquisition, its value has to be estimated for each value of $k$ when dealing with the non-Cartesian case. This can be performed using the power iterative method. ${ }^{35}$

Note that when $\boldsymbol{\Psi}$ defines an orthonormal basis, we get || $\boldsymbol{\Psi} \mid \|=1$. Nevertheless, SubProblem (5) makes use of an analysis-based prior which enables the use of over-complete dictionaries and guarantees enhanced image quality at reconstruction at the cost of longer reconstruction times. ${ }^{23,36}$

\subsection{Multi-channel reception coil}

A multi-channel acquisition refers to the usage of multi-receiver phased array to collect the MR data. It is well-established that the use of such coil allows an increase of the input SNR. ${ }^{37}$ In what follows, we denote by $L$ the number of channels in this kind of coils. In this context, the k-space measurements associated with $\Omega_{k}$ collected by the $\ell^{\text {th }}$ channel are defined as $\boldsymbol{y}_{\Omega_{k}, \ell} \in \mathbb{C}^{k C}$. All collected $\mathrm{k}$-space data are then stacked in $\boldsymbol{Y}_{\Omega_{k}}=\left[\boldsymbol{y}_{\Omega_{k}, 1}, \ldots, \boldsymbol{y}_{\Omega_{k}, L}\right] \in \mathbb{C}^{k C \times L}$.

In the literature, reconstruction methods for multi-channel acquisition can be split in two classes. The first one models the coil sensitivity maps $\left(\boldsymbol{S}_{\ell}\right)_{1 \leq \ell \leq L}$ where $\boldsymbol{S}_{\ell} \in \mathbb{C}^{N \times N}$ represents the $\ell^{\text {th }}$ channel sensitivity profile. In these techniques, a single image is recovered from the combination of all channels. ${ }^{14,23}$ The reconstruction problem is often formulated as follows:

$$
\hat{\boldsymbol{x}}=\underset{\boldsymbol{x} \in \mathbb{C}^{N}}{\arg \min } \frac{1}{2} \sum_{\ell=1}^{L}\left\|\boldsymbol{F}_{\Omega_{S}} \boldsymbol{S}_{\ell} \boldsymbol{x}-\boldsymbol{y}_{\Omega_{S}, \ell}\right\|_{2}^{2}+\lambda\|\boldsymbol{\Psi} \boldsymbol{x}\|_{1}, \quad \lambda>0 .
$$

However, the coil sensitivities depend on the scanned subject, therefore they have to be calibrated for each subject. The calibration step can be performed beforehand ${ }^{38,39}$ and then some estimates $\left(\widehat{\boldsymbol{S}}_{\ell}\right)_{1 \leq \ell \leq L}$ can be injected in Eq. (6). Alternatively, the reconstruction can be viewed as a blind bilinear inverse problem ${ }^{40}$ where the optimization alternates between sensitivity profile estimation and image recovery steps. While the second approach is more computationally demanding, the first one does not really fit the constraints of online reconstruction. Indeed, as any shot is able to entirely cover the center of k-space, low frequency information associated with spatially smooth sensitivity profiles cannot be extracted in a straight manner. Moreover, a second weakness of this formalism lies in the fact that the gradient Lipschitz constant $\beta_{k}$ depends on the coil sensitivities and cannot be computed in advance if we assume that no calibration scan has been run beforehand.

The second set of approaches falls in the class of calibrationless methods, which by definition do not require any prior knowledge of sensitivity profiles. As a consequence, they try to reconstruct an image per channel with some regularizing constraints across channels to impose consistency. Either the regularization is performed in the kspace domain where a low-rank penalty is applied to a Hankel matrix, ${ }^{41-43}$ or it is imposed in a sparse transformed domain for instance in order to promote group sparsity. ${ }^{16,44,45}$ While k-space-based methods demonstrate good image recovery for Cartesian sampling ${ }^{41}$, their application to non-Cartesian trajectories implies a gridding step such as GROG $^{46}$ that strongly degrades the final image quality. For that reason, domain-based calibrationless reconstruction seems better suited to online processing of multi-channel non-Cartesian k-space data.

For the aforementioned reasons, we will use a domain-based calibrationless formulation that reads as follows:

$$
\widehat{\boldsymbol{X}}=\underset{\boldsymbol{X} \in \mathbb{C}^{N \times L}}{\arg \min } \frac{1}{2}\left\|\boldsymbol{F}_{\Omega_{S}} \boldsymbol{X}-\boldsymbol{Y}_{\Omega_{S}}\right\|_{2}^{2}+\lambda g(\boldsymbol{\Psi} \boldsymbol{X}), \quad \lambda>0 .
$$

In Eq. (7) $g$ refers to a structured sparsity promoting term. As was shown recently, ${ }^{16}$ this formulation is able to recover images with a quality competitive with state-of-the art methods, ${ }^{40}$ especially for non-Cartesian sampling schemes. Subsequently, the Octagonal Shrinkage and Clustering Algorithm for Regression (OSCAR) ${ }^{16}$ based calibrationless reconstruction method is implemented, where the wavelet transform $\boldsymbol{\Psi}$ decomposes the stack of images into a stack of coefficients $\boldsymbol{C}=\boldsymbol{\Psi} \boldsymbol{X}=\left[\boldsymbol{c}_{1}, \ldots, \boldsymbol{c}_{L}\right]=\left[\boldsymbol{\Psi} \boldsymbol{x}_{1}, \ldots, \boldsymbol{\Psi} \boldsymbol{x}_{L}\right] \in \mathbb{C}^{N_{\Psi} \times L}$. Each vector of wavelet 
coefficients $\boldsymbol{c}_{\ell}$ with $\ell \in\{1, \ldots, L\}$ is made up of $B$ bands. The $b \in\{1, \ldots, B\}$ band contains $P_{b}$ coefficients $\left(c_{\ell, b, j}\right)_{1 \leq j \leq P_{b}}$. The OSCAR penalty is expressed as follows:

$$
g_{\mathrm{OSCAR}}(\boldsymbol{C})=\sum_{b=1}^{B} \sum_{j=1}^{P_{b}}\left(\sum_{\ell=1}^{L}\left|c_{\ell, b, j}\right|+\gamma \sum_{\ell^{\prime}<\ell} \max \left\{\left|c_{\ell, b, j}\right|,\left|c_{\ell^{\prime}, b, j}\right|\right), \quad \gamma>0 .\right.
$$

Here, we can introduce the straightforward extension of our online formulation to the matrix case. The online calibrationless reconstruction Subproblem (3) thus reads:

$$
(\forall k \in\{1, \ldots, S\}) \quad \hat{\boldsymbol{X}}_{k}=\underset{\boldsymbol{X} \in \mathbb{C}^{N \times L}}{\arg \min } \frac{S}{2 k}\left\|\boldsymbol{F}_{\Omega_{k}} \boldsymbol{X}-\boldsymbol{Y}_{\Omega_{k}}\right\|_{2}^{2}+\lambda g_{\mathrm{OSCAR}}(\boldsymbol{\Psi} \boldsymbol{X}) .
$$

On the one hand, the gradient Lipschitz constant $\beta_{k}$ only depends on the sampling scheme $\Omega_{k}$, hence it can be computed offline and loaded at the beginning of each scan. Noticeably, changing the size $b_{s}$ of mini-batches impacts the values of $\left(\beta_{k}\right)_{1 \leq k \leq S}$. On the other hand, the proximity operator of $g_{\mathrm{OSCAR}}$ is explicit and can be computed efficiently [47, Eq. 24].

The performance of this new multi-channel image reconstruction formulation and its ability to comply with online processing constraints will be discussed in the next section.

\section{PARAMETERS SETTING \& RESULTS}

\subsection{Parameters setting}

General MR Parameters. Two baboon and human ex vivo brains were scanned on a 7 Tesla MR system (Siemens Healthineers, Erlangen, Germany). The motivation for ex vivo imaging was the absence of motion. The acquisition parameters were set as follows: $\mathrm{TR}=550 \mathrm{~ms}$ (for collecting 11 slices), $\mathrm{TE}=30 \mathrm{~ms}$ and flip angle $\mathrm{FA}=25^{\circ}$ with an in-plane resolution of $400 \mu \mathrm{m}$ for a field of view (FOV) of $20.4 \mathrm{~cm}$ which leads to a matrix size of $N=512 \times 512$.

For both single and multi-channel acquisitions, a fully sampled Cartesian reference scan was collected. It was composed of 512 lines with 512 samples each. The image was reconstructed using FFT and used as reference for computing image quality scores.

Single-channel acquisition parameters. The baboon brain was scanned using a birdcage $1 \mathrm{Tx} / 1 \mathrm{Rx}$ coil and the acquisition parameters defined hereabove. To maintain a high SNR, we considered a slice thickness of $3 \mathrm{~mm}$. All animal studies were conducted in accordance with the European convention for animal care and the NIHs Guide for the Care and Use of Laboratory Animals. Fig. 1 shows (a) the Cartesian reference, (b) the retrospective Cartesian variable density sampling mask and (c) the prospective 15 -fold accelerated non-Cartesian Sparkling ${ }^{10}$ pattern. Here, we remind that retrospective under-sampling was performed by applying the sampling mask to the already collected fully sampled data whereas prospective under-sampling collects less data directly and may be contaminated by additional artifacts due for instance to discrepancy between the prescribed and actual sampling trajectories. Prospective validation is thus more challenging.

The Cartesian mask (Fig. 1(b)) was composed of $S=176$ lines of $C=512$ samples each, leading to UF $=$ $\mathrm{AF}=2.9$. The segmented acquisition was ordered in time by considering that central lines of $\mathrm{k}$-space were collected first and then that others were acquired in a random order. The Sparkling sampling pattern (Fig. 1(c)) was generated with $S=34$ shots from a radial initialization, each shot being composed of $C=3072$ samples leading to $\mathrm{UF}=2.5$ and $\mathrm{AF}=15$. The total scan time using Sparkling was reduced to $18.7 \mathrm{~s}$ instead of $281.6 \mathrm{~s}$ for the fully sampled Cartesian reference. The acquisition was segmented in time according to the golden-angle ordering scheme, as it was proven ${ }^{48}$ to increase the maximize the k-space coverage during the scan. Hence, between two consecutive shots a rotation of $111^{\circ}$ was applied.

Multi-channel acquisition parameters. An ex vivo human brain was scanned using a $1 \mathrm{Tx} / 32 \mathrm{Rx}$ (Nova Medical Inc., Washington, MA, USA), i.e. $L=32$ with a slice thickness of $1.5 \mathrm{~mm}$. The donor gave his 
(a)

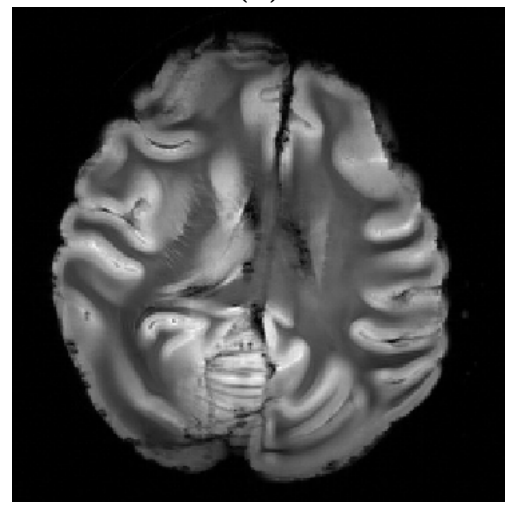

(b)

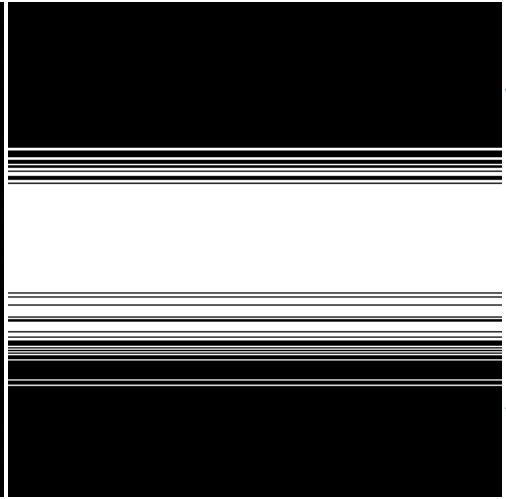

(c)

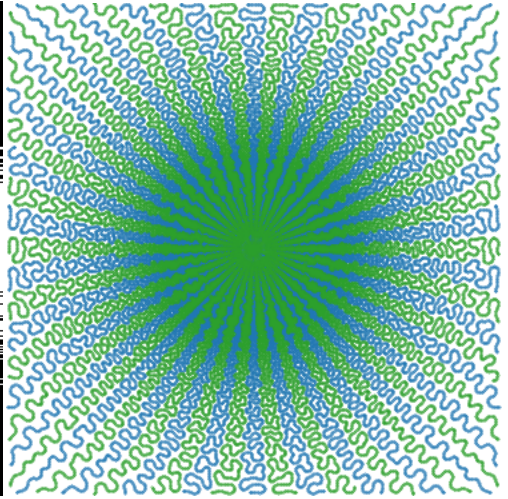

Figure 1: Single-channel coil acquisition: (a) ex vivo baboon brain Cartesian reference image, (b) retrospective Cartesian under-sampling scheme $(\mathrm{AF}=\mathrm{UF}=2.9),(\mathbf{c})$ prospective non-Cartesian Sparkling sampling scheme $(\mathrm{AF}=15, \mathrm{UF}=2.5)$.

written consent before death to the donation program of University of Tours, France. The reference slice is displayed in Fig. 2(a). Prospectively accelerated Sparkling acquisitions were considered with AF $=8$ and $\mathrm{AF}=15$ corresponding to $S=64$ and $S=34$ shots, respectively. Both are shown in Fig. 2(b)-(c). The 15-fold accelerated sampling pattern was the same as the one used in single-channel acquisition. We also investigated less accelerated acquisition $(\mathrm{AF}=8)$ using radially-initialized Sparkling trajectories to see to what extent this may impact online reconstruction. In that case, the total scan time was $35.2 \mathrm{~s}$ instead of $281.6 \mathrm{~s}$ for the Cartesian reference. A larger number of shots actually offers more degrees of freedom to define the mini-batch size $b_{s}$ in online reconstruction.

(a)

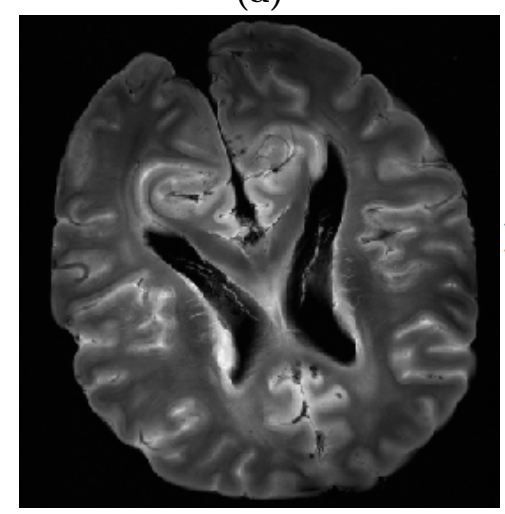

(b)

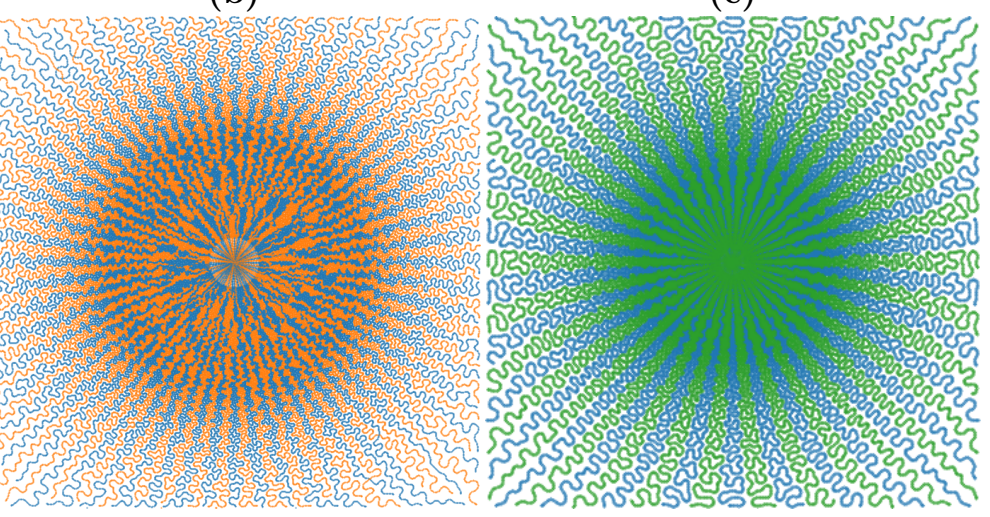

Figure 2: Multi-channel coil acquisition: (a) ex vivo human brain Cartesian reference image, (b) prospective Sparkling sampling pattern with $S=64$ spokes (i.e. $\mathrm{AF}=8$ ) and $C=3072$ points (i.e. $\mathrm{UF}=1.3$ ), (c) prospective Sparkling sampling pattern with $S=34$ spokes (i.e. AF $=15$ ), each one being composed of $C=3072$ points (i.e. $\mathrm{UF}=2.5$ ).

Reconstruction parameters. The decimated Symmlet 8 Wavelet Transform with 4 decomposition scales was used as sparsifying transform. Regarding hyper-parameter $\lambda$ in Eq. (5) and the pair $(\lambda, \gamma)$ in Eq. (9), they have been set retrospectively so as to maximize the structural similarity score (SSIM) ${ }^{49}$ to the reference image once all data are available (i.e. offline scenario). Note that in a more clinically plausible setting, at least hyper-parameter $\lambda$ needs either to be estimated using the noise statistical properties ${ }^{50}$ or set using cross-validation across slices in $2 \mathrm{D}$ imaging. ${ }^{51}$ Also, it might decrease over mini-batches as far as information provided by the data gets 
more reliable. For non-Cartesian Fourier acquisition the GPU ${ }^{52}$ implementation of the NUFFT ${ }^{11}$ was used. In Section 3.2, we compare the performances of several batch sizes with a given number of iterations defined by Eq. (4). Once all shots are considered in Algorithm 1, i.e. $k=S$, then the number of iterations is set to $n_{b}=200$ to guarantee that convergence is reached.

Computing parameters setting. All experiments were run on a machine with 128 GB of RAM and an 8-core $(2.40 \mathrm{GHz})$ Intel Xeon E5-2630 v3 Processor. All the codes have been developed in Python using the PySAP package*. The values of computing times have been obtained using 5 epochs of 10 Condat-Vú iterations each, the mean and standard deviation being summarized in Tab. 1. Using multiple epochs permits to account for potential variability in computing times due to concomitant processes running on the machine. Although the NUFFT is usually slower than the FFT, here we observed the converse as we used the GPU implementation of the NUFFT and the CPU implementation of the FFT. A more fair comparison might be achieved using the GPU version of FFT but this was not required for the present online study.

Table 1: Computing time for one iteration estimated on 5 epochs of 10 iterations each using timeit.

\begin{tabular}{|c|c|c|}
\hline Cartesian single-channel & Non-Cartesian single-channel & Non-Cartesian multi-channel \\
\hline $93.9 \mathrm{~ms} \pm 14.4 \mathrm{~ms}$ & $78.2 \mathrm{~ms} \pm 8.9 \mathrm{~ms}$ & $4.29 \mathrm{~s} \pm 111 \mathrm{~ms}$ \\
\hline
\end{tabular}

\subsection{Results}

Cartesian sampling for single-channel coil acquisition. First we implemented the online reconstruction pipeline for single-channel acquisition using the retrospectively under-sampled Cartesian k-space mask shown in Fig. 1(b). Mini-batches of increasing size $\left(b_{s} \in\{1,4,16,22,44,88\}\right)$ were tested against the offline reconstruction scenario $\left(b_{s}=176\right)$. In Fig. 3(a)-(b), we show the evolution over time of the global cost function and the SSIM score. The time origin corresponds to the beginning of the scan. All settings eventually converge to the same value both in terms of cost function and SSIM score. This confirms that the final image is the same. Moreover Fig. 4(top-row) depicts partial reconstructions for the tested mini-batch sizes by the end of acquisition (i.e. before taking into account the last mini-batch). While large mini-batches show aliasing artifacts (see Fig. 4 for $b_{s}=44$, and $\left.b_{s}=88\right)$, the small batches deliver pretty accurate images, which tends to demonstrate the benefits of using small batch sizes for online reconstruction purposes. The reason for which we observed aliasing artifacts in large batch sizes is the varying amount of available k-space data in the top row of Fig. 4 . Indeed, by the end of the acquisition (i.e. before the process of the last mini-batch at $T=\mathrm{TR}-\epsilon$ ) only a single shot is missing for $b_{s}=1$ while for larger $b_{s}$ (such as for $b_{s}=88$ ) a large number of shots are missing in the data-fidelity term (when $b_{s}=88$ half of the spoke are not processed).

Non-Cartesian sampling for single-channel coil acquisition. The non-Cartesian case was also tested using 15-fold accelerated Sparkling trajectories as shown in Fig. 1(c). As this sampling pattern comprises $S=34$ shots, the only possible tunings of the mini-batch size $b_{s}$ are its factors $1,2,17$. In that context, the GPU implementation of the NUFFT was really helpful to comply with online reconstruction constraints and maintain a short time per iteration as it allows us to iterate over a sufficiently large number of iterations in each subproblem and to explore small batch sizes too. We respectively used $n_{b}=8,16,24$ for increasing batch sizes $b_{s}=1,2,17$. Fig. 5 demonstrates even more clearly than in Cartesian sampling that the evolution over time of both the global cost function and the SSIM score benefit from small batch sizes to reach almost convergence by the end of acquisition (depicted in dotted black line). The fastest converging online scenario corresponds to $b_{s}=1$ (red trace in Fig. 5) as $n_{b}=8$ are sufficient to significantly decrease the cost function. This is confirmed by the partial solutions obtained by the end of acquisition (see Fig. 6). The reconstructed images for $b_{s}=1$ or $b_{s}=2$ are already very close to the Cartesian reference as compared to the one obtained for the larger batch size $b_{s}=17$. Once again, the aliasing artifacts shown in Fig. 6(top-row) for $b_{s}=17$ are due to a larger part of missing data (half in this case).

These results confirm the feasibility of online MR image reconstruction from non-Cartesian k-space data in a single-channel coil acquisition scenario.

\footnotetext{
${ }^{*}$ https://github.com/CEA-COSMIC/pysap
} 
(a)

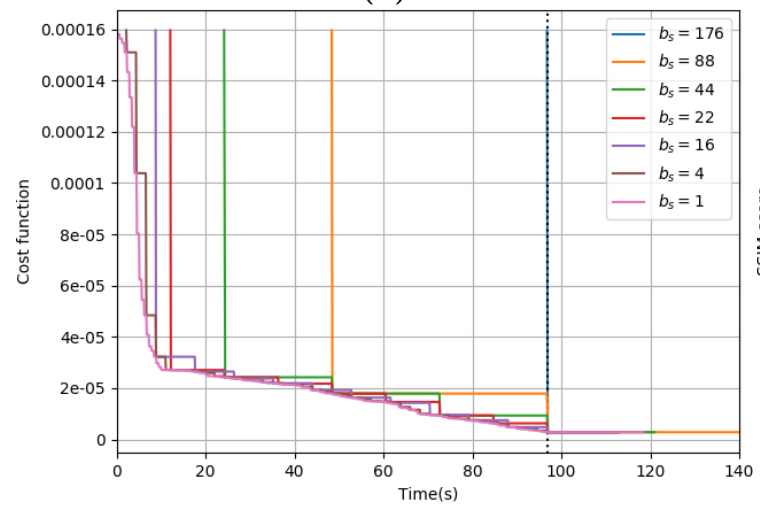

(b)

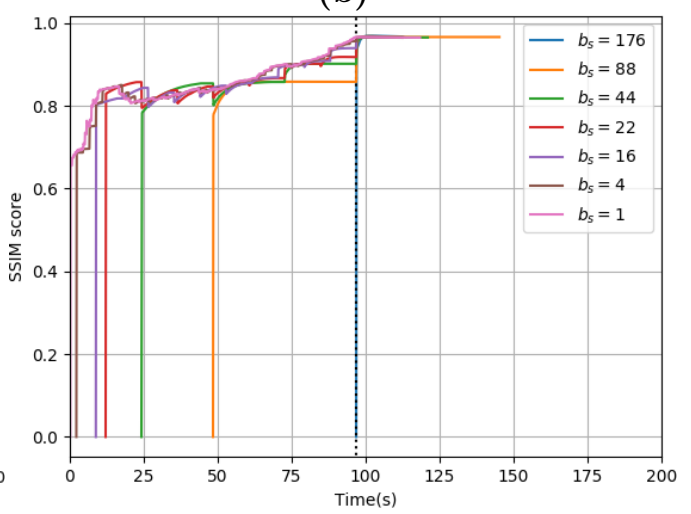

Figure 3: Single-channel retrospective Cartesian reconstruction: (a) evolution over time of cost function in in Eq. (5) and of (b) the SSIM score for different batch sizes $b_{s}$. The dark dashed line marks the end of acquisition.
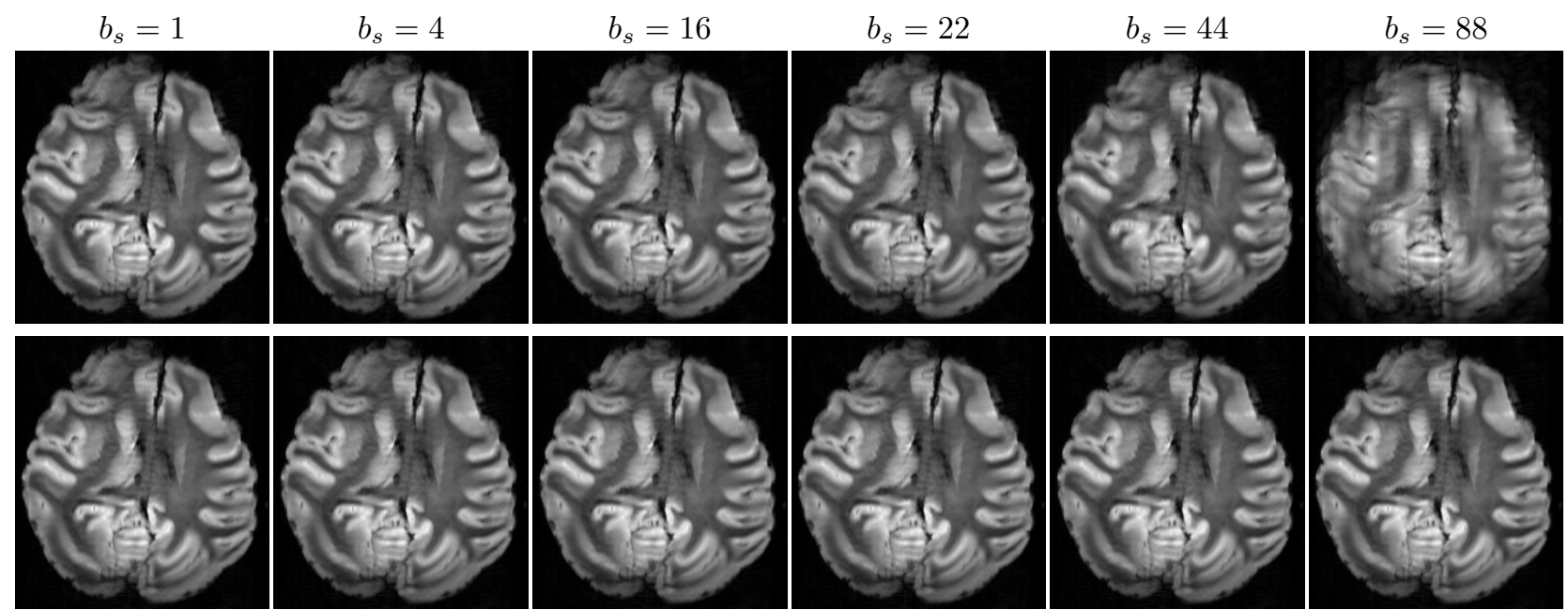

Figure 4: Online reconstruction of 15 -fold ( $S=34$ shots) retrospectively accelerated Cartesian scan of ex vivo baboon brain in a single-channel coil acquisition setup. Images reconstructed for increasing values of mini-batch size $b_{s}$ at the end of acquisition (top) and at convergence (bottom).

Non-Cartesian sampling for multi-channel coil acquisition. As multi-channel online reconstruction is more demanding from a computational viewpoint, the way constraint (4) can be satisfied requires a larger number of shots. Because Algorithm 1 assumes uniform batch sizes over iterations, we decided to explore a drastically less accelerated (i.e. 8-fold) Sparkling acquisition in which $S=64$ are collected. This allows us to get more flexibility in the setting of the batch size $b_{s}$ as compared to the 15-fold accelerated Sparkling sampling scheme where $S=34$ can be split only in $b_{s}=2$ or $b_{s}=17$.

Fig. 7 shows that there is only a little advantage for small batch sizes (i.e. $b_{s}=8$ and $b_{s}=16$ ) in the parallel imaging framework. This results from the fact that the overall computing time per iteration and constraint (4) allow to perform only $n_{b}=1\left(n_{b}=2\right.$, respectively) iteration(s) when $s_{b}=8\left(s_{b}=16\right.$, respectively). The zoom in the curve of SSIM score in Fig. 7(b) confirms this tiny advantage for small batch sizes. Stronger evidence in favor of small values of $b_{s}$ is shown in Fig. 8(top) where the ventricles (especially the right one) appear darker in $b_{s}=8$ as compared to $b_{s}=32$. Images obtained at convergence (Fig. 8 (bottom)), i.e. once $n_{b}=200$ iterations have been run from $k=S$ mini-batches, confirm the convergence to the Cartesian reference.

Overall, in this multi-channel acquisition scenario the main bottleneck remains the computation cost per iteration. With a diminished cost, we could perform a larger number of iterations in particular for small batch 
(a)

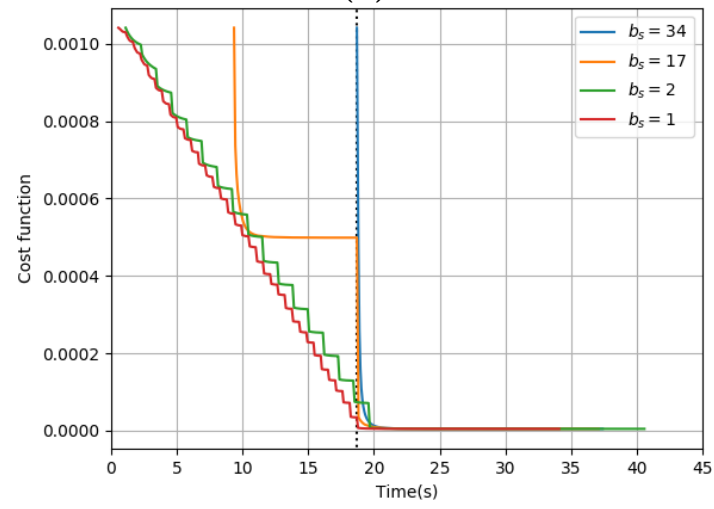

(b)

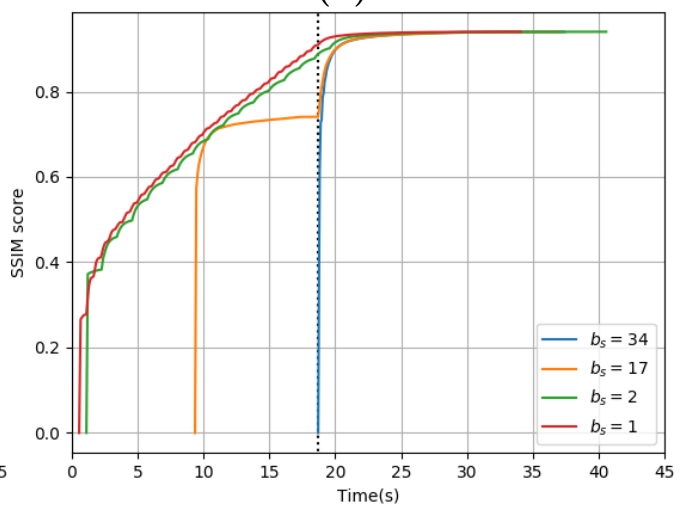

Figure 5: Online reconstruction of 15-fold ( $S=34$ shots) prospectively accelerated Sparkling scan of ex vivo baboon brain in a single-channel coil acquisition setup. Evolution over time of (a) the cost function in Eq. (5), and of (b) the SSIM score for batch sizes $b_{s}$ corresponding to the primary factors of $S$. The dark dashed line marks the end of acquisition.
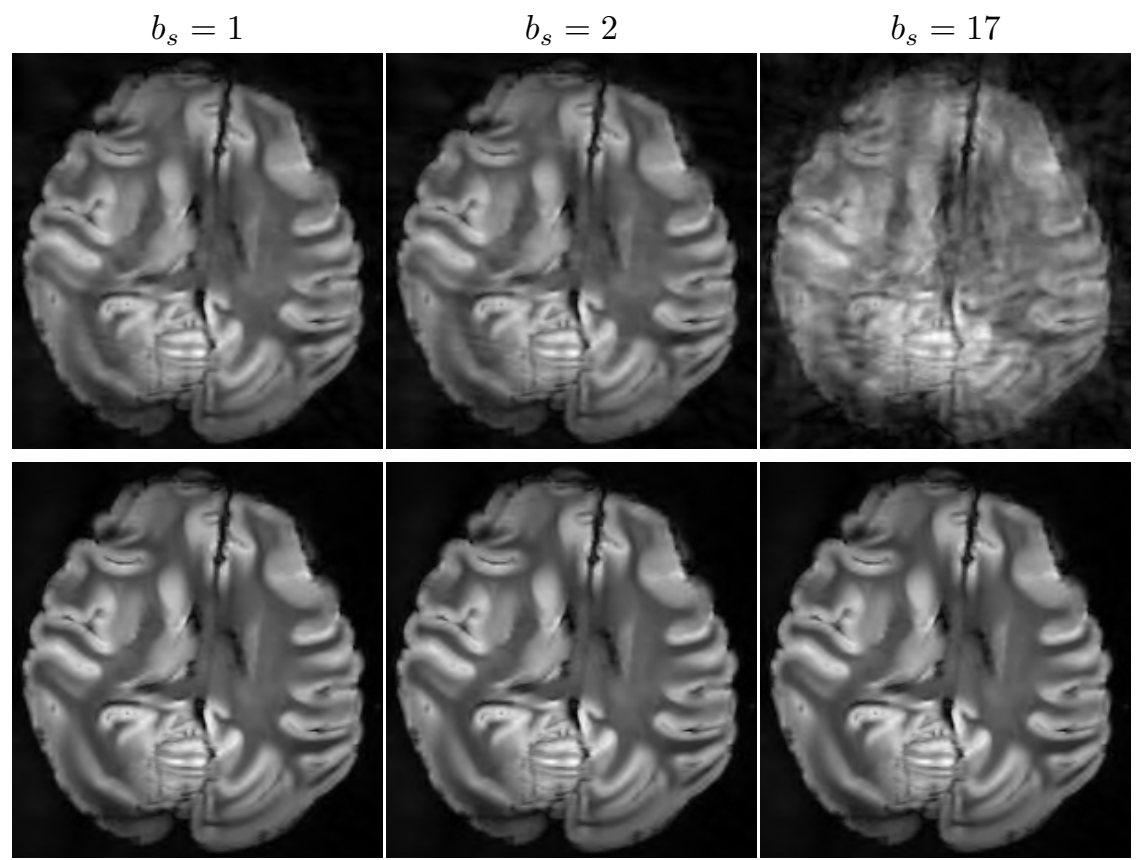

Figure 6: Online reconstruction of 15 -fold ( $S=34$ shots) prospectively accelerated Sparkling scan of ex vivo baboon brain in a single-channel coil acquisition setup. Images reconstructed for increasing values of mini-batch size $b_{s}$ by the end of acquisition (top) and at convergence (bottom).

sizes (for instance $n_{b}=4$ for $b_{s}=8$ ) and get better MR images by the end of acquisition.

Last, we investigated a Sparkling acquisition scenario corresponding to the same sampling pattern (Fig. 2(c)) as the one studied in the single-channel setup $(S=34,15$-fold acceleration). In this setup, the online reconstruction approach is not really viable as shown first in Fig. 9: a single mini-batch configuration $\left(b_{s}=17\right)$ was explored given the timing constraint and it does not converge by the end of acquisition both in terms of cost function and SSIM score as only $n_{b}=2$ iterations were carried out. This observation is then confirmed on the reconstructed MR image reported in Fig. 10(a), which is severely corrupted by aliasing artifacts. Nevertheless, the final solution at convergence (Fig. 10(b)) exactly matches the Cartesian reference. 
(a)

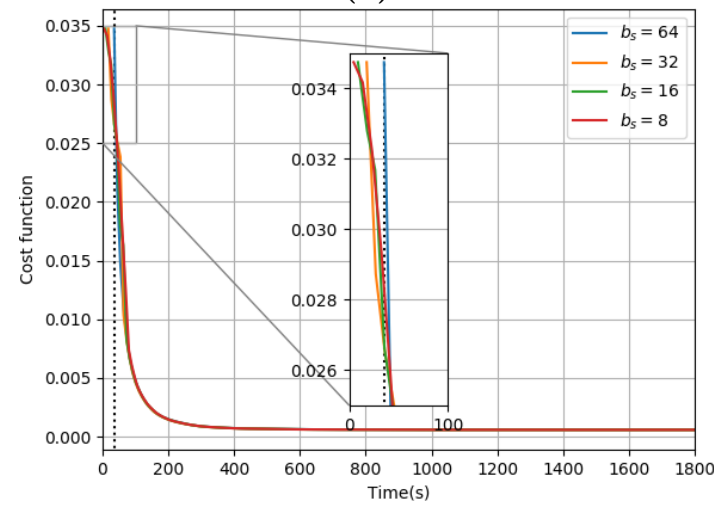

(b)

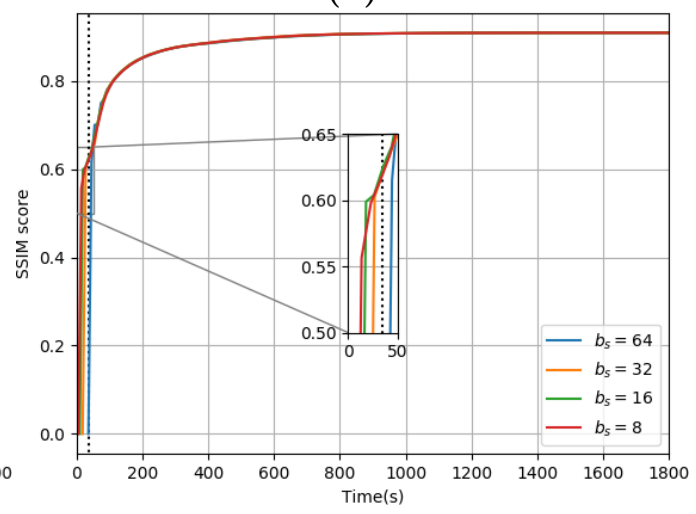

Figure 7: Online reconstruction of 8-fold ( $S=64$ shots) prospectively accelerated Sparkling scan of ex vivo human brain in a multi-channel coil acquisition setup. Evolution over time of (a) the cost function in Eq. (9) and of (b) the SSIM score for increasing values of batch size $b_{s}$. The dark dashed line marks the end of acquisition.
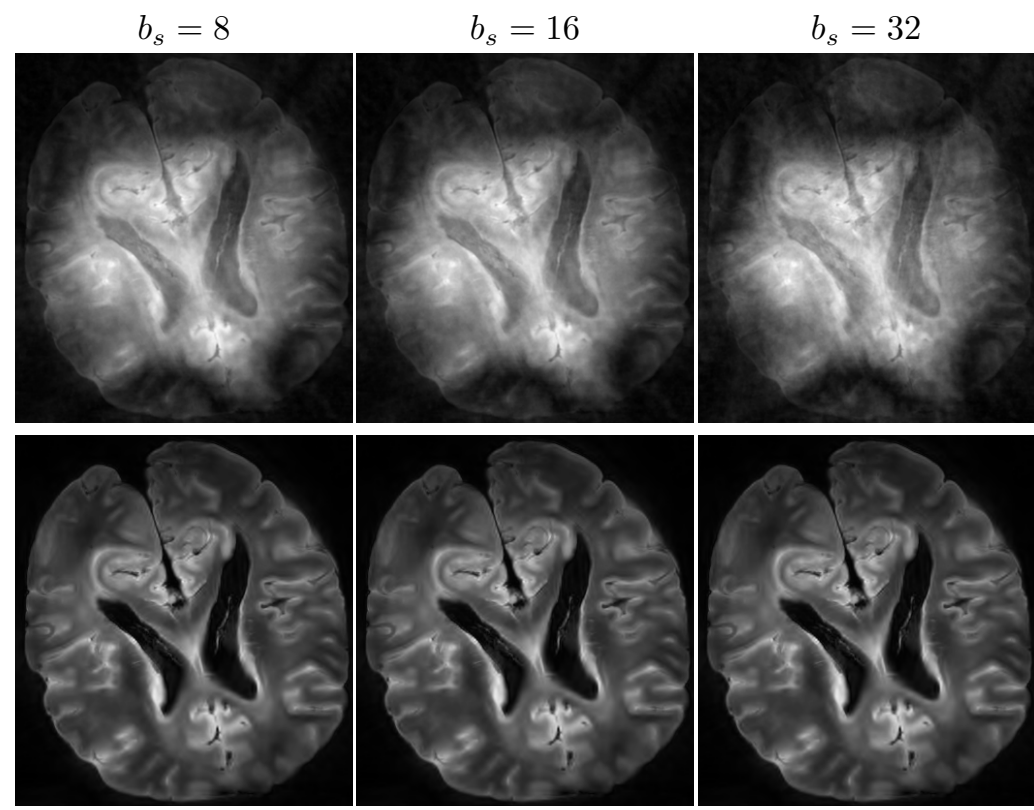

Figure 8: Online reconstruction of 8-fold $(S=64$ shots) prospectively accelerated Sparkling scan of ex vivo human brain in a multi-channel coil acquisition setup. Images reconstructed for increasing values of mini-batch size $b_{s}$ by the end of acquisition (top) and at convergence (bottom).

\section{DISCUSSION \& CONCLUSION}

Acceleration of MRI acquisition has been a major area of research for the last decade, especially in the highresolution context where motion becomes critical. In this context, $\mathrm{k}$-space data is collected in a segmented manner through multiple consecutive shots separated by times of repetition to allow the recovery of the magnetization. Among the different acceleration strategies developed so far, Compressed Sensing is the most appealing as it enables larger acceleration factors without degrading the image quality at the reconstruction step. However, the price to pay lies in a long iterative process for image reconstruction.

While most of the recent research activity in accelerated MRI reconstruction has moved to deep learning approaches (see ${ }^{53-56}$ to cite a few), requiring large data sets which may be unavailable in the high-resolution context, in this work we explored a radically different approach, namely online CS MR image reconstruction. 
(a)

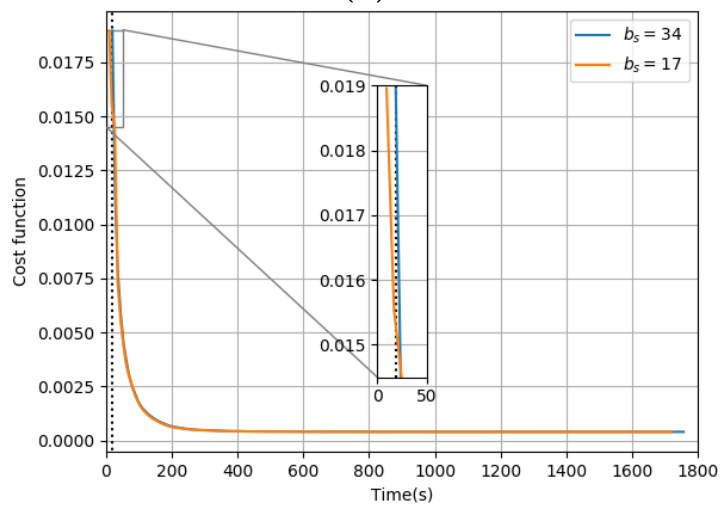

(b)

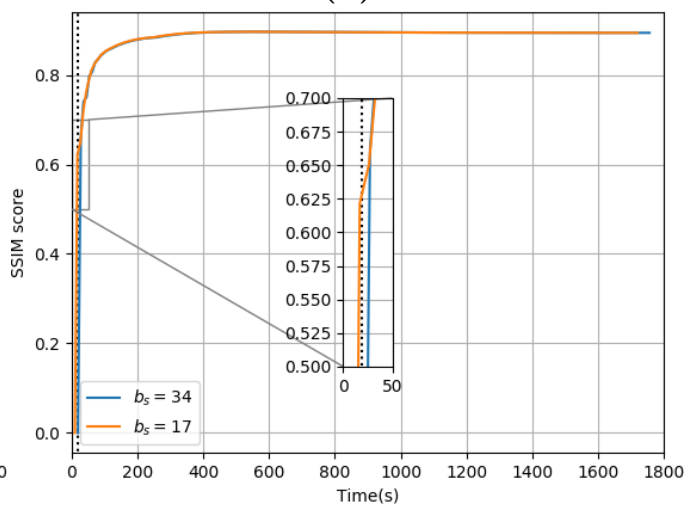

Figure 9: Online reconstruction of 15-fold ( $S=34$ shots) prospectively accelerated Sparkling scan of ex vivo human brain in a multi-channel coil acquisition setup. Evolution over time of (a) the cost function in Eq. (9) and of (b) the SSIM score for batch sizes $b_{s}=17$ and $b_{s}=34$. The dark dashed line marks the end of acquisition.

(a)

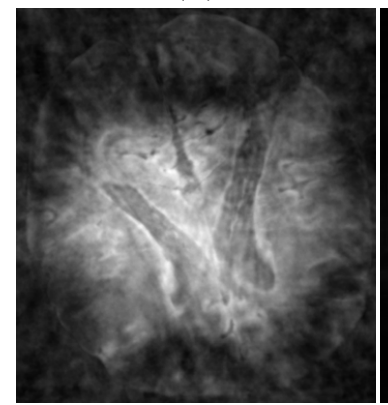

(b)

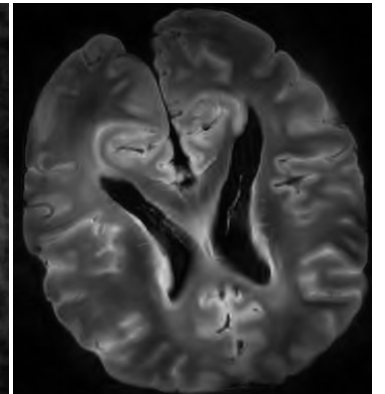

Figure 10: Online reconstruction of 15 -fold $(S=34$ shots) prospectively accelerated Sparkling scan of ex vivo human brain in a multi-channel coil acquisition setup using $b_{s}=17$. (a) Partial solution obtained by the end of acquisition and (b) at convergence.

We applied the proposed methodology to $\mathrm{T}_{2}^{*}$-weighted imaging as this contrast offers a good fit to online timing requirements and insightful information on the brain tissues at 7 Tesla.

Our online reconstruction approach relies on a mini-batch formulation which consists in splitting the acquired shots in multiple subsets and aggregating them over time through a mini-batch processing. We take advantage of the period of time between mini-batches to launch the reconstruction from incomplete data. The use of a warmrestart mechanism to set up new variables in a given mini-batch reconstruction from the solution obtained at the previous iteration allowed us to save computing time and converge faster. Still in this context, CS reconstruction was formulated as the minimization of a regularized criterion that combines a data consistency term and a sparsity promoting penalization. Although the $\ell_{1}$-norm term is the most straightforward in CS reconstruction, it only allows to address the single-channel reconstruction scenario. A second contribution of this paper was thus to extend the online framework to parallel imaging reconstruction in which k-space data are collected over multiple channels. For this purpose we made use of a recently proposed calibrationless method $^{16}$ and promoted group sparsity across channels using OSCAR-norm regularization. This calibrationless formulation presents the advantage of being well-suited to non-Cartesian sampling. Also, it does not require prior knowledge on the sensitivity profiles associated with the multiple channels. Importantly, the proposed online approach relies on convex optimization and its convergence to the global solution is guaranteed once all mini-batches have been treated.

The proposed framework was validated on single and multi-channel acquisitions at 7 Tesla. We considered ex vivo imaging to avoid any motion-related concern. In the single-channel acquisition setup, a retrospective Cartesian sampling was implemented using variable density over phase encoding lines with an acceleration factor 
of 2.9. When the shots are split in small batch sizes, the online reconstruction showed a significant gain in terms of speedup as the SSIM score of the image solution computed by the end of acquisition was very close to that of the final image delivered by offline reconstruction. This finding also holds for non-Cartesian acquisitions performed with a single-channel coil.

However, online reconstruction gets more challenging in the parallel imaging setup as both the computing time per iteration and memory usage increase. Hence, it becomes harder to comply with the timing constraints using small batch sizes without decreasing the number of iterations per mini-batch processing significantly. We still observed some advantages for MR online reconstruction in 8-fold accelerated Sparkling acquisitions using a 32-receiver coil. In contrast, in highly accelerated acquisitions (i.e. 15-fold Sparkling) we showed that the current approach is not able to recover artifact-free images by the end of acquisition as only large batch sizes can be managed in this acquisition scenario. This conclusion calls for exploring new directions to overcome this difficulty. In what follows, we briefly describe three of them, which are left for perspectives.

A first guess for improving numerical efficiency of online reconstruction would consist of improving the initialization $\boldsymbol{x}_{0}$ using a density compensated regridded solution. ${ }^{57}$ A second direction is related to the computational complexity of minimizing the cost function in Eq. (9). In, ${ }^{16}$ we actually studied the separate cost per iteration of computing the proximity operator of OSCAR-norm regularization and the gradient of the data consistency term. As computing the proximity operator is much more expensive, we could start by minimizing the data consistency term over the first mini-batches before injecting the regularizing term when aggregating the last mini-batch in the online processing. This would help alleviate the computational burden and enlarge the number of iterations during mini-batch processing. A last direction that could be relevant to explore is related to coil compression. With the current trend of enlarging the number of channels in reception coils in order to continuously improve the input SNR (e.g. 64 channel Nova coil available on 3 Tesla systems), coil compression could drastically limit memory usage and computing time. ${ }^{58}$ However, the question of optimal combination of coil compression with calibrationless reconstruction is still an open issue.

Overall, this novel framework enables to reduce the reconstruction times in CS MRI applications. It is compatible with the Gadgetron implementation ${ }^{15}$ that enables to embed those reconstruction into the scanner and to send back decent images to the scanner console during the course of the scan. This may be really helpful to guide the physician for selecting further pulse MR sequences in order to sharpen his medical diagnosis.

Future work will be devoted to the adaptation of this framework to other imaging contrasts (e.g., $\mathrm{T}_{2}$-weighted imaging) and pulse sequences (e.g., turbo spin echo) for which larger times of repetition (e.g. 5 s) give us the opportunity to successfully apply the proposed approach in the multi-channel acquisition setting.

Acknowledgements. We would like to thank Dr Antoine Grigis (CEA/NeuroSpin) and Dr Samuel Farrens (CEA/IRFU, CosmoStat) for their significant contribution to the development of PySAP software in which the present work has been implemented. This work was mainly supported by a 2016 DRF Impulsion grant (COSMIC, P.I.: P.C.) and a 2016 France Life Imaging grant (Multi-CSMRI) between the different partners. It was also partially supported by the Institut Universitaire de France and by the Agence Nationale de la Recherche under grants ANR-17-CE40-0004-01 MAJIC.

\section{REFERENCES}

[1] F. Fazekas, R. Kleinert, G. Roob, G. Kleinert, P. Kapeller, R. Schmidt, and H.-P. Hartung, "Histopathologic analysis of foci of signal loss on gradient-echo $\mathrm{t} 2{ }^{*}$-weighted $\mathrm{mr}$ images in patients with spontaneous intracerebral hemorrhage: evidence of microangiopathy-related microbleeds," American Journal of Neuroradiology 20(4), pp. 637-642, 1999.

[2] F. Bloch, "Nuclear induction," Phys. Rev. 70, pp. 460-474, Oct 1946.

[3] D. A. Feinberg, J. Hale, J. Watts, L. Kaufman, and A. Mark, "Halving mr imaging time by conjugation: demonstration at $3.5 \mathrm{~kg} ., "$ Radiology 161(2), pp. 527-531, 1986.

[4] K. Pruessmann, P. Weiger, M. Scheidegger, and P. Boesiger, "SENSE: sensitivity encoding for fast MRI," Magnetic Resonance in Medicine 42(5), pp. 952-962, 1999.

[5] M. A. Griswold, P. M. Jakob, R. M. Heidemann, M. Nittka, V. Jellus, J. Wang, B. Kiefer, and A. Haase, "Generalized autocalibrating partially parallel acquisitions (grappa)," Magnetic Resonance in Medicine: An Official Journal of the International Society for Magnetic Resonance in Medicine 47(6), pp. 1202-1210, 2002. 
[6] F. A. Breuer, M. Blaimer, M. F. Mueller, N. Seiberlich, R. M. Heidemann, M. A. Griswold, and P. M. Jakob, "Controlled aliasing in volumetric parallel imaging (2d caipirinha)," Magnetic Resonance in Medicine: An Official Journal of the International Society for Magnetic Resonance in Medicine 55(3), pp. 549-556, 2006.

[7] D. Donoho, "Compressed sensing," IEEE Transactions on Information Theory 52(4), pp. 1289-1306, 2006.

[8] M. Lustig, D. Donoho, and J. Pauly, "Sparse MRI: The application of compressed sensing for rapid MR imaging," Magnetic Resonance in Medicine 58(6), pp. 1182-1195, 2007.

[9] J. H. Lee, B. A. Hargreaves, B. S. Hu, and D. G. Nishimura, "Fast 3D imaging using variable-density spiral trajectories with applications to limb perfusion," Magnetic Resonance in Medicine 50(6), pp. 1276-1285, 2003.

[10] C. Lazarus, P. Weiss, N. Chauffert, F. Mauconduit, L. El Gueddari, C. Destrieux, I. Zemmoura, A. Vignaud, and P. Ciuciu, "Sparkling: variable-density k-space filling curves for accelerated t $2{ }^{*}$-weighted mri," Magnetic resonance in medicine, 2019.

[11] J. Fessler and B. Sutton, "Nonuniform fast Fourier transforms using min-max interpolation," 2003.

[12] J. Keiner, S. Kunis, and D. Potts, "Using NFFT 3-a software library for various nonequispaced fast fourier transforms," ACM Transactions on Mathematical Software (TOMS) 36(4), p. 19, 2009.

[13] M. Guerquin-Kern, M. Haberlin, K. P. Pruessmann, and M. Unser, "A fast wavelet-based reconstruction method for magnetic resonance imaging," IEEE transactions on medical imaging 30(9), pp. 1649-1660, 2011.

[14] L. Chaâri, J.-C. Pesquet, A. Benazza-Benyahia, and P. Ciuciu, "A wavelet-based regularized reconstruction algorithm for sense parallel mri with applications to neuroimaging," Medical image analysis 15(2), pp. 185-201, 2011.

[15] M. Hansen and T. Srensen, "Gadgetron: An open source framework for medical image reconstruction," Magnetic Resonance in Medicine 69(6), pp. 1768-1776.

[16] L. El Gueddari, P. Ciuciu, E. Chouzenoux, A. Vignaud, and J.-C. Pesquet, "Calibrationless oscar-based image reconstruction in compressed sensing parallel mri.," in IEEE International Symposium of Biomedical Imaging, 2019.

[17] J.-J. Moreau, "Proximité et dualité dans un espace hilbertien," Bulletin de la Société mathématique de France $\mathbf{9 3}$ pp. 273-299, 1965.

[18] M. A. Bernstein, K. F. King, and X. J. Zhou, Handbook of MRI pulse sequences, Elsevier, 2004.

[19] G. Puy, P. Vandergheynst, and Y. Wiaux, "On variable density compressive sampling," IEEE Signal Processing Letters 18(10), pp. 595-598, 2011.

[20] N. Chauffert, P. Ciuciu, J. Kahn, and P. Weiss, "Variable density sampling with continuous trajectories. Application to MRI," SIAM Journal on Imaging Sciences 7, pp. 1962-1992, Nov. 2014.

[21] C. Boyer, N. Chauffert, P. Ciuciu, J. Kahn, and P. Weiss, "On the generation of sampling schemes for Magnetic Resonance Imaging," SIAM Journal on Imaging Sciences 9(4), pp. 2039-2072, 2016.

[22] N. Pustelnik, A. Benazza-Benhayia, Y. Zheng, and J.-C. Pesquet, "Wavelet-based image deconvolution and reconstruction," Wiley Encyclopedia of Electrical and Electronics Engineering, pp. 1-34, 1999.

[23] A. Florescu, E. Chouzenoux, J.-C. Pesquet, P. Ciuciu, and S. Ciochina, "A majorize-minimize memory gradient method for complex-valued inverse problems," Signal Processing 103, pp. 285-295, Jan 2014.

[24] P. L. Combettes and J.-C. Pesquet, "Proximal splitting methods in signal processing," in Fixed-point algorithms for inverse problems in science and engineering, pp. 185-212, Springer, 2011.

[25] I. Daubechies, M. Defrise, and C. De Mol, "An iterative thresholding algorithm for linear inverse problems with a sparsity constraint," Communications on Pure and Applied Mathematics: A Journal Issued by the Courant Institute of Mathematical Sciences 57(11), pp. 1413-1457, 2004.

[26] A. Beck and M. Teboulle, "A fast iterative shrinkage-thresholding algorithm for linear inverse problems," SIAM journal on imaging sciences 2 (1), pp. 183-202, 2009.

[27] A. B. Taylor, J. M. Hendrickx, and F. Glineur, "Exact worst-case performance of first-order methods for composite convex optimization," SIAM Journal on Optimization 27(3), pp. 1283-1313, 2017.

[28] P. L. Combettes and J.-C. Pesquet, "Stochastic approximations and perturbations in forward-backward splitting for monotone operators," Pure and Applied Functional Analysis 1(1), pp. 13-37, 2016.

[29] J. Eckstein and D. P. Bertsekas, "On the douglasrachford splitting method and the proximal point algorithm for maximal monotone operators," Mathematical Programming 55(1-3), pp. 293-318, 1992.

[30] A. Chambolle and T. Pock, "A first-order primal-dual algorithm for convex problems with applications to imaging," Journal of mathematical imaging and vision 40(1), pp. 120-145, 2011.

[31] L. Condat, "A primal-dual splitting method for convex optimization involving Lipschitzian, proximable and linear composite terms," Journal of Optimization Theory and Applications 158(2), pp. 460-479, 2013.

$[32]$ B. Vũ, "A splitting algorithm for dual monotone inclusions involving cocoercive operators," Advances in Computational Mathematics 38, pp. 667-681, Apr 2013

[33] M. Elad, P. Milanfar, and R. Rubinstein, "Analysis versus synthesis in signal priors," Inverse problems 23(3), p. 947, 2007.

[34] L. Condat, "A primal-dual splitting method for convex optimization involving lipschitzian, proximable and linear composite terms," Journal of Optimization Theory and Applications 158, pp. 460-479, Aug 2013. 
[35] W. Press, S. Teukolsky, W. Vetterling, and B. Flannery, Numerical Recipes 3rd Edition: The Art of Scientific Computing. Cambridge University Press, New York, NY, USA, 3 ed., 2007.

[36] H. Cherkaoui, L. El Gueddari, C. Lazarus, A. Grigis, F. Poupon, A. Vignaud, S. Farrens, J. Starck, and P. Ciuciu, "Analysis vs synthesis-based regularization for combined compressed sensing and parallel MRI reconstruction at 7 tesla," in 26th European Signal Processing Conference (EUSIPCO 2018), 2018.

[37] P. Roemer, W. Edelstein, C. Hayes, S. Souza, and O. Mueller, "The NMR phased array," Magnetic resonance in medicine 16(2), pp. 192-225, 1990.

[38] M. Uecker, P. Lai, M. Murphy, P. Virtue, M. Elad, J. Pauly, S. Vasanawala, and M. Lustig, "ESPIRiT-an eigenvalue approach to autocalibrating parallel MRI: where SENSE meets GRAPPA," Magnetic Resonance in Medicine 71(3), pp. 990-1001, 2014.

[39] L. El Gueddari, C. Lazarus, H. Carrié, A. Vignaud, and P. Ciuciu, "Self-calibrating nonlinear reconstruction algorithms for variable density sampling and parallel reception MRI," in 10th IEEE SAM workshop, 2018.

[40] H. She, R. Chen, D. Liang, E. DiBella, and L. Ying, "Sparse BLIP: Blind iterative parallel imaging reconstruction using compressed sensing," Magnetic Resonance in Medicine 71(2), pp. 645-660, 2014.

[41] J. Haldar, "Low-rank modeling of local $k$-space neighborhoods (LORAKS) for Constrained MRI," IEEE transactions on Medical Imaging 33(3), pp. 668-681, 2014.

[42] P. J. Shin, P. E. Larson, M. A. Ohliger, M. Elad, J. M. Pauly, D. B. Vigneron, and M. Lustig, "Calibrationless parallel imaging reconstruction based on structured low-rank matrix completion," Magnetic resonance in medicine 72(4), pp. 959-970, 2014.

[43] D. Lee, K. H. Jin, E. Y. Kim, S.-H. Park, and J. C. Ye, "Acceleration of mr parameter mapping using annihilating filter-based low rank hankel matrix (aloha)," Magnetic resonance in medicine 76(6), pp. 1848-1864, 2016.

[44] A. Majumdar and R. Ward, "Calibration-less multi-coil MR image reconstruction," Magnetic Resonance in Medicine 30(7), pp. 1032-1045, 2012.

[45] J. Trzasko and A. Manduca, "Calibrationless parallel MRI using CLEAR," in Signals, Systems and Computers (ASILOMAR), 2011 Conference Record of the Forty Fifth Asilomar Conference on, pp. 75-79, IEEE, 2011.

[46] N. Seiberlich, F. A. Breuer, M. Blaimer, K. Barkauskas, P. Jakob, and M. Griswold, "Non-cartesian data reconstruction using GRAPPA operator gridding (GROG)," Magnetic Resonance in Medicine 58(6), pp. 1257-1265, 2007.

[47] X. Zeng and M. Figueiredo, "The ordered weighted 11 norm: Atomic formulation, projections, and algorithms," arXiv preprint arXiv:1409.4271, 2014.

[48] S. Winkelmann, T. Schaeffter, T. Koehler, H. Eggers, and O. Doessel, "An optimal radial profile order based on the golden ratio for time-resolved mri," IEEE transactions on medical imaging 26(1), pp. 68-76, 2006.

[49] Z. Wang, A. Bovik, H. Sheikh, and E. Simoncelli, "Image quality assessment: from error visibility to structural similarity," IEEE transactions on Image Processing 13(4), pp. 600-612, 2004.

[50] C. M. Stein, "Estimation of the mean of a multivariate normal distribution," The annals of Statistics , pp. 1135-1151, 1981.

[51] T. Hastie, R. Tibshirani, J. Friedman, and J. Franklin, "The elements of statistical learning: data mining, inference and prediction," The Mathematical Intelligencer 27(2), pp. 83-85, 2005.

[52] J.-M. Lin, "Python non-uniform fast Fourier transform (PyNUFFT): An accelerated non-Cartesian MRI package on a heterogeneous platform (CPU/GPU)," Journal of Imaging 4(3), p. 51, 2018.

[53] J. Sun, H. Li, Z. Xu, et al., "Deep admm-net for compressive sensing MRI," in Advances in neural information processing systems, pp. 10-18, 2016.

[54] M. Mardani, E. Gong, J. Y. Cheng, S. S. Vasanawala, G. Zaharchuk, L. Xing, and J. M. Pauly, "Deep generative adversarial neural networks for compressive sensing MRI," IEEE transactions on medical imaging 38(1), pp. 167-179, 2018.

[55] K. Hammernik, T. Klatzer, E. Kobler, M. P. Recht, D. K. Sodickson, T. Pock, and F. Knoll, "Learning a variational network for reconstruction of accelerated MRI data," Magnetic resonance in medicine 79(6), pp. 3055-3071, 2018.

[56] B. Zhu, J. Z. Liu, S. F. Cauley, B. R. Rosen, and M. S. Rosen, "Image reconstruction by domain-transform manifold learning," Nature 555(7697), p. 487, 2018.

[57] J. A. Fessler and D. C. Noll, "Iterative reconstruction methods for non-cartesian mri," in Proc. ISMRM Workshop on Non-Cartesian MRI, 2007.

[58] M. Buehrer, K. P. Pruessmann, P. Boesiger, and S. Kozerke, "Array compression for mri with large coil arrays," Magnetic Resonance in Medicine: An Official Journal of the International Society for Magnetic Resonance in Medicine 57(6), pp. 1131-1139, 2007. 